\title{
Effects of different oral care strategies on postoperative pneumonia in infants with mechanical ventilation after cardiac surgery: a prospective randomized controlled study
}

\author{
Xian-Rong $\mathbf{Y u}^{1,2}$, Ning $\mathrm{Xu}^{1,2}$, Shu-Ting Huang ${ }^{1,2}$, Qi-Liang Zhang ${ }^{1,2}$, Zeng-Chun Wang ${ }^{1,2,3}$, Hua Cao ${ }^{1,2}$, \\ Qiang Chen ${ }^{1,2,3}$
}

${ }^{1}$ Department of Cardiac Surgery, Fujian Maternity and Child Health Hospital, Affiliated Hospital of Fujian Medical University, Fuzhou, China; ${ }^{2}$ Fujian Key Laboratory of Women and Children's Critical Diseases Research, Fujian Maternity and Child Health Hospital, Fuzhou, China; ${ }^{3}$ Department of Cardiovascular Surgery, Union Hospital, Fujian Medical University, Fuzhou, China

Contributions: (I) Conception and design: XR Yu, Q Chen; (II) Administrative support: H Cao, ZC Wang; (III) Provision of study materials or patients: N Xu, ST Huang; (IV) Collection and assembly of data: QL Zhang, Q Chen; (V) Data analysis and interpretation: Q Chen, XR Yu; (VI) Manuscript writing: All authors; (VII) Final approval of manuscript: All authors.

Correspondence to: Qiang Chen, MD. Department of Cardiac Surgery, Fujian Maternity and Child Health Hospital, Affiliated Hospital of Fujian Medical University, No. 18, Daoshan Road, Gulou District, Fuzhou 350001, China. Email: chenqiang2228@163.com.

\begin{abstract}
Background: To explore the effects of different oral care strategies on postoperative pneumonia in infants with mechanical ventilation after cardiac surgery.

Methods: A prospective randomized controlled study was conducted at a hospital in Fujian Province, China. Participants were randomly divided into the breast milk oral care group, physiological saline oral care group, and sodium bicarbonate oral care group to explore the effects of different oral care strategies on postoperative pneumonia in infants on mechanical ventilation cardiac surgery.

Results: The mechanical ventilation duration, the hospitalization costs, and the length of intensive care unit (ICU) stay and postoperative hospital stay in the breast milk oral care group were significantly shorter than those in the physiological saline oral care group and the sodium bicarbonate oral care group. The incidence of postoperative pneumonia in the breast milk oral care group was $3.2 \%$, which was significantly lower than that in the physiological saline oral care group $(22.6 \%)$ and the sodium bicarbonate oral care group (19.4\%).

Conclusions: Using breast milk for oral care in infants after cardiac surgery has a lower incidence of postoperative pneumonia than traditional oral care strategies of physiological saline and sodium bicarbonate, and it is worthy of clinical application.
\end{abstract}

Keywords: Oral care; infant; congenital heart defect (CHD)

Submitted Sep 16, 2020. Accepted for publication Dec 15, 2020.

doi: $10.21037 / \mathrm{tp}-20-295$

View this article at: http://dx.doi.org/10.21037/tp-20-295

\section{Introduction}

Postoperative pneumonia is the most common cause of hospital-acquired infections in the intensive care unit (ICU), with an incidence of 8-28\% (1-3). Postoperative pneumonia will result in a longer mechanical ventilation duration, a more extended hospital stay, higher hospitalization costs, and increased mortality (4-8). Many studies have shown that oropharyngeal bacterial colonization plays a vital role in the pathogenesis of postoperative pneumonia $(9,10)$. Tracheal intubation not only damaged the natural barrier between the oropharynx and the trachea but also provided a direct and rapid way for bacteria to enter the lower respiratory tract, which would cause the occurrence of postoperative pneumonia $(11,12)$. 
Due to the presence of intracardiac malformations and abnormal blood flow, the nutritional status and physique of infants with congenital heart disease are often worse before surgery. Postoperative pneumonia incidence is higher after withstanding cardiac surgery injury, cardiopulmonary bypass injury, myocardial ischemiareperfusion injury, prolonged endotracheal intubation, and mechanical ventilation (13). Oral care is one of the critical aspects of postoperative rehabilitation measures, improving patients' health, and preventing severe complications such as pneumonia (14). Numerous studies have shown that proper oral care can reduce the colonization of potentially pathogenic bacteria in the oropharynx and reduce the occurrence of postoperative pneumonia $(15,16)$. However, there are few reports on the practice of oral care for infants after cardiac surgery. In this study, a prospective randomized controlled study was conducted to evaluate the effect of different oral care strategies on postoperative pneumonia in infants on mechanical ventilation after cardiac surgery. We present the following article in accordance with the CONSORT checklist (available at http://dx.doi. org/10.21037/tp-20-295).

\section{Methods}

\section{Study design and definition}

The present study was approved by the ethics committee of Fujian Maternity and Child Health Hospital (No. 2020YJ181) and adhered to the tenets of the Declaration of Helsinki (as revised in 2013). A prospective, randomized, controlled study was conducted in the cardiac ICU at a provincial hospital. The clinical data of 93 cases of infantile patients who underwent cardiac surgery using cardiopulmonary bypass in our hospital from January 2020 to June 2020 were collected. Signed informed consent was taken from the patients' parents/guardians. The inclusion criteria were as follows: (I) infants after cardiac surgery with cardiopulmonary bypass; (II) the patient's anatomical treatment was satisfactory, cardiac function recovered well after the operation, and hemodynamics was stable. The exclusion criteria were as follows: (I) infants with severe liver, kidney, and other essential organ dysfunctions; (II) parents of patients who refused to participate in this study.

The perioperative antibiotic prevention strategies we adopted were as follows: cefmetazole was given intravenously $30 \mathrm{~min}$ before the operation, and another dose was given if the operation time was longer than three hours, and one dose was given within 24 hours after the operation according to the prescription formula. The diagnosis of postoperative pneumonia was mainly based on manifestations of fever, wheezing, cough, shortness of breath, wet rales auscultated on chest examination, and radiographic abnormalities such as spotty or patchy infiltration in a chest radiograph (17). Bacterial culture of airway secretions was helpful to identify pathogens and assist treatment. Generally, on the second day after the infants developed pneumonia-related symptoms or signs, we combined with biochemical indicators, imaging, and other examinations to diagnose postoperative pneumonia. This usually happened on the second postoperative day.

The mechanical ventilation mode adopted in this study was synchronized intermittent mandatory ventilation (SIMV). Our initial ventilator settings were set as follows: $\mathrm{FiO}_{2}$ regulated from 0.21 to 0.60 , PIP regulated from 15 to $20 \mathrm{cmH}_{2} \mathrm{O}$, RR regulated from 20 to 30 times/min, and PEEP regulated from 2 to $6 \mathrm{cmH}_{2} \mathrm{O}$, and then the parameters were adjusted according to the infant's pulmonary condition and arterial blood gas results.

Routine clinical data were recorded and analyzed. Besides, postoperative mechanical ventilation duration, the length of ICU stay, the length of hospital stay, hospitalization costs, bacterial culture of the respiratory tract, and postoperative related complications were also statistically analyzed. Postoperative inflammatory indicators, such as white blood cell (WBC), C-reactive protein (CRP), and procalcitonin (PCT) were also recorded.

\section{Calculation of the study sample size}

Based on the incidence of postoperative pneumonia between the three groups in the pre-experiment, assuming the difference between the three independent populations was $10 \%, \alpha=0.05$, and $\beta=0.2$, the number of participants needed was 28 in each group. Assuming a $10 \%$ attrition rate, the total sample size was 93 (31 per group).

According to the different oral care strategies used, the individuals were divided into three groups: the breast milk oral care group, the physiological saline oral care group, and the sodium bicarbonate oral care group. The researchers randomly divided eligible parents into three groups based on computer-generated random numbers.

\section{Intervention methods}

Before implementing oral care, the oral cavity retentate 
Table 1 Comparison of general data among the three groups

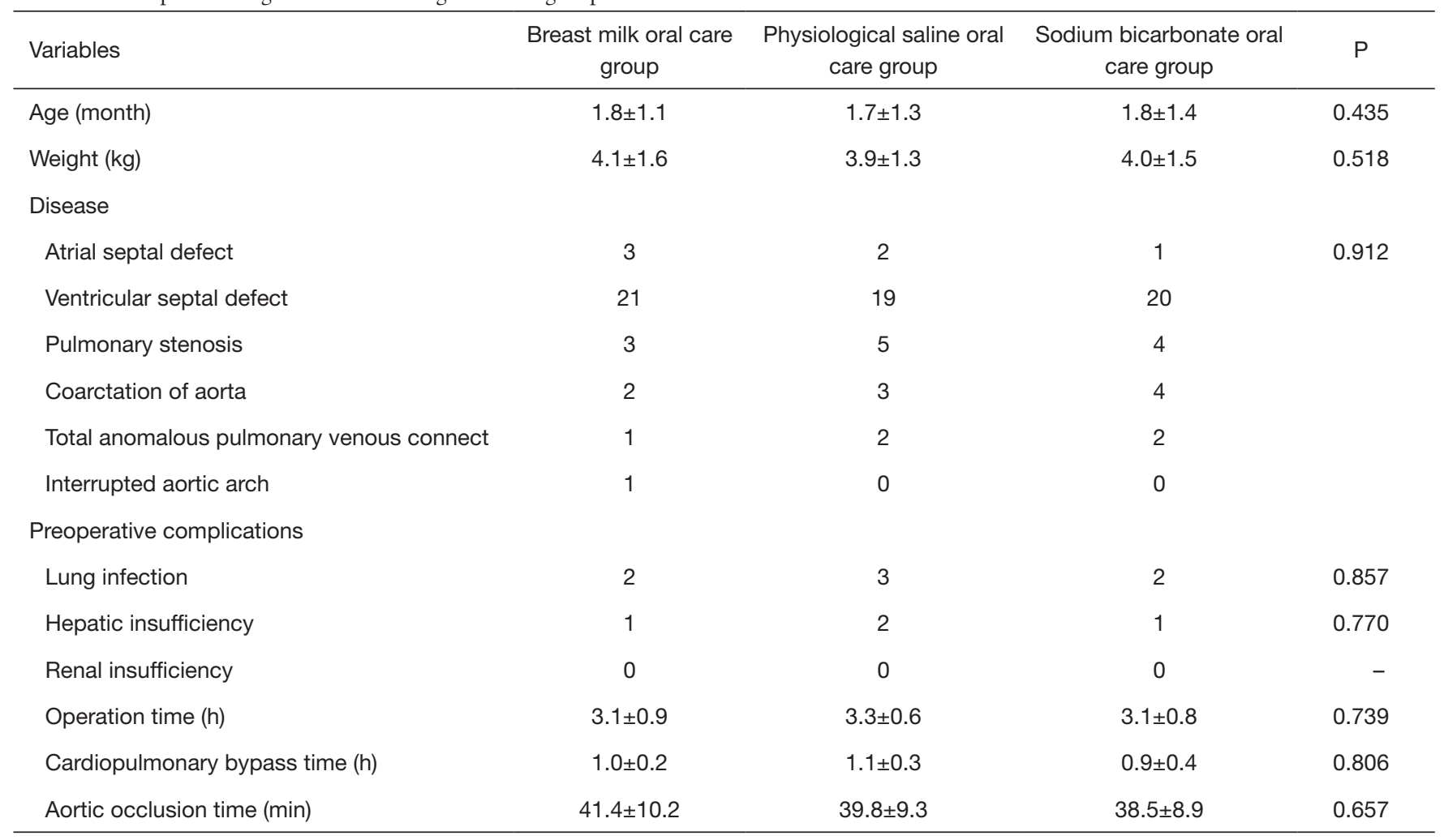

h, hour; min, minute.

and the subglottic cavity were removed using negative pressure. According to different oral care strategies and the corresponding groups, breast milk, physiological saline, and sodium bicarbonate were used for oral care. The different oral care products were used to scrub the wall of the lip, cheek, gingiva, hard palate, tongue surface, sublingual, and tracheal intubation in turn, and each part was scrubbed with at least one cotton swab for at least 30 seconds. Oral care was performed every 3 hours. The researcher screened eligible parents for the study and collected relevant clinical data.

\section{Statistical analysis}

SPSS19.0 software was used to perform statistical analysis in this study. Continuous data are presented as the mean \pm standard deviation and range. ANOVA was used to compare the mean of the three groups, and the S-N-K(S) method was used to compare each pair. The $\chi^{2}$ or Fisher's test was used to categorize the variables. A $\mathrm{P}$ value of $<0.05$ was defined as statistically significant.

\section{Results}

Comparison of the general patient information, such as age (1.8 \pm 1.1 vs. $1.7 \pm 1.3$ vs. $1.8 \pm 1.4$ months), weight $(4.1 \pm 1.6$ vs. $3.9 \pm 1.3$ vs. $4.0 \pm 1.5 \mathrm{~kg})$, operation time $(3.1 \pm 0.9$ vs. $3.3 \pm 0.6$ vs. $3.1 \pm 0.8 \mathrm{~h})$, cardiopulmonary bypass time $(1.0 \pm 0.2 v s$. $1.1 \pm 0.3$ vs. $0.9 \pm 0.4 \mathrm{~h})$, and aortic occlusion time $(41.4 \pm 10.2$ vs. $39.8 \pm 9.3$ vs. $38.5 \pm 8.9 \mathrm{~min})$, in the three groups, was not statistically significant $(\mathrm{P}>0.05)$, which showed that the three groups of patients were homogeneous and comparable (Table 1). Deep hypothermic circulatory arrest (DHCA) was used in $15.4 \%$ of cases, with the arrest time of $30.5 \pm 17.8$ minutes and the average minimum body temperature of $28.1 \pm 0.5^{\circ} \mathrm{C}$. The minimal core body temperature of patients who did not use DHCA was $32.5 \pm 0.6^{\circ} \mathrm{C}$.

Compared with the physiological saline oral care group and the sodium bicarbonate oral care group, the mechanical ventilation duration $(3.6 \pm 1.1 v s .4 .9 \pm 1.8 v s .4 .7 \pm 1.7 \mathrm{~h})$, the length of intensive care time $(5.8 \pm 1.8 v s .7 .8 \pm 2.7$ vs. $7.9 \pm$ $3.1 \mathrm{~d})$, hospitalization costs $(5.3 \pm 1.1$ vs. $7.8 \pm 1.5$ vs. $7.6 \pm 1.7$ $10,000 \mathrm{CNY}$ ) and the length of postoperative hospital stay $(13.3 \pm 2.1$ vs. $16.8 \pm 3.9$ vs. $17.0 \pm 4.0 \mathrm{~d})$ were significantly 
Table 2 Comparison of postoperative conditions among the three groups

\begin{tabular}{lccc}
\hline \multirow{2}{*}{ Variables } & \multirow{2}{*}{$\begin{array}{c}\text { Breast milk oral care group } \\
\text { Mechanical ventilation duration (d) }\end{array}$} & $\begin{array}{c}\text { Physiological saline oral care } \\
\text { group }\end{array}$ & $\begin{array}{c}\text { Sodium bicarbonate oral care } \\
\text { group }\end{array}$ \\
\hline The length of ICU stay (d) & $3.6 \pm 1.1$ & $4.9 \pm 1.8^{*}$ & $4.7 \pm 1.7^{\star}$ \\
Hospitalization costs (10,000 CNY) & $5.8 \pm 1.8$ & $7.8 \pm 2.7^{\star}$ & $7.9 \pm 3.1^{\star}$ \\
The length of hospital stay (d) & $5.3 \pm 1.1$ & $7.8 \pm 1.5^{\star}$ & 0.028 \\
\hline
\end{tabular}

*, indicated $\mathrm{P}<0.05$ compared with breast milk oral care group; ICU, intensive care unit; CNY, Chinese Yuan.

Table 3 Comparison of postoperative complications among the three groups

\begin{tabular}{|c|c|c|c|c|}
\hline Variables & Breast milk oral care group & Physiological saline oral care group & $\begin{array}{l}\text { Sodium bicarbonate oral } \\
\text { care group }\end{array}$ & $\mathrm{P}$ \\
\hline Postoperative pneumonia & 1 & $7^{*}$ & $6^{\star}$ & 0.031 \\
\hline Arrhythmia & 3 & 4 & 4 & 0.902 \\
\hline Hepatic insufficiency & 4 & 5 & 3 & 0.750 \\
\hline Poor wound healing & 1 & 2 & 2 & 0.809 \\
\hline
\end{tabular}

*, indicated $\mathrm{P}<0.05$ compared with breast milk oral care group.

shorter in the breast milk oral care group $(\mathrm{P}<0.05)$. There was no significant difference between the physiological saline oral care group and the sodium bicarbonate oral care group (Table 2).

The incidence of postoperative pneumonia in the breast milk oral care group was $3.2 \%$, and it was significantly lower than that in the physiological saline oral care group $(22.6 \%)$ and the sodium bicarbonate oral care group (19.4\%) $(\mathrm{P}=0.031)$. There were 4 cases $(28.6 \%)$ with positive sputum culture in the lower respiratory tract in infants with postoperative pneumonia, among which 3 cases were in the physiological saline oral care group, including 2 cases of Escherichia coli and 1 case of Klebsiella pneumonia. There was 1 case of Enterobacter cloacae in the sodium bicarbonate oral care group. The treatment of postoperative pneumonia mainly adopted comprehensive treatment, including active anti-infection and other symptomatic support treatment. There was no statistically significant difference in the incidence of postoperative low cardiac output syndrome, arrhythmia, liver dysfunction, renal dysfunction, and poor wound healing after surgery in the three groups (Table 3).

In comparing postoperative inflammatory markers in the three groups, there was no statistically significant difference in the WBC, CRP, and PCT levels one day after surgery $(\mathrm{P}>0.05)$. The CRP level in the breast milk oral care group three days after surgery was lower than those in the other two groups $(\mathrm{P}<0.05)$. Besides, the WBC, CRP, and PCT levels in the breast milk oral care group were all lower than those in the other two groups five days after surgery $(\mathrm{P}<0.05)$ (Table 4).

\section{Discussion}

Congenital heart disease is one of the most common congenital structural deformities, and approximately $2-3 \%$ of newborns have congenital heart disease (18). For some infants with congenital heart disease, early cardiac surgery needs to be performed under hypothermia and cardiopulmonary bypass, which is often accompanied by cardiopulmonary dysfunction after surgery. As a result, those infantile patients need a relatively long period of mechanically assisted ventilation to support cardiopulmonary function and improve their circulatory status. Because the infant's immune function is relatively low, the pulmonary function is not fully developed. A series of pathophysiological changes produced by cardiac 
Table 4 Postoperative inflammatory markers between the three groups

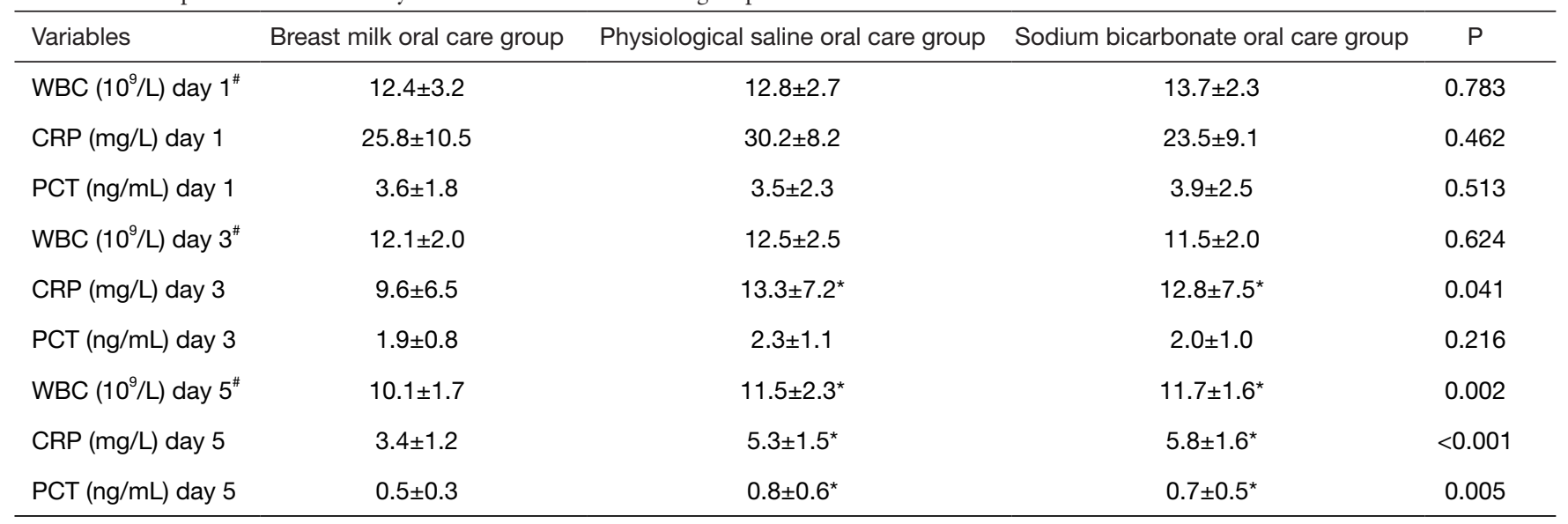

\#, postoperative days; *, indicated $\mathrm{P}<0.05$ compared with the breast milk oral care group. WBC, white blood cell; CRP, C-reactive protein; PCT, procalcitonin.

malformation, surgical trauma, and cardiopulmonary bypass injury make infants on mechanical ventilation more likely to develop postoperative pneumonia after cardiac surgery (13).

It is generally believed that oral hygiene is directly related to postoperative pneumonia because oral bacteriology characteristics in patients with mechanical ventilation are different from those in ordinary people and nonmechanically ventilated patients $(19,20)$. There are more than ten kinds of bacteria living in the oral cavity of the average population, and they are in dynamic equilibrium, which will not cause disease in the physiological state. However, due to endotracheal intubation and mechanical ventilation, patients' oral cavity and airway on mechanical ventilation are continuously opening. Besides, these patients are forbidden to drink. The oral cavity's self-cleaning ability and the oral hygiene environment status are reduced, resulting in a break in the dynamic balance of the oral cavity and significant colonization with bacteria. Then, the oral bacteria move down the tube and causes postoperative pneumonia $(21,22)$. Messika et al. conducted a study and found that the incidence of ventilator-associated pneumonia in patients with colonized bacteria in the oropharynx was significantly higher than in the patients without colonized bacteria (23). An increasing number of scholars at home and abroad have studied the prevention methods of postoperative pneumonia in recent years, among which oral care is considered one of the most important measures to prevent postoperative pneumonia. It has become a central issue in clinical nursing research (24). The oral care strategies are varied, and the studies on its different effects are increasing to find the best oral care strategies. To find more suitable oral care strategies for infants after cardiac surgery, we conducted this study to explore the effects of different oral care strategies on postoperative pneumonia in infants on mechanical ventilation.

The commonly used oral care strategies are physiological saline, sterilized injectable water, sodium bicarbonate solution, and so on, and the nursing effect of various solutions is different. It is essential for infants with prolonged endotracheal intubation and mechanical ventilation after cardiac surgery to explore the suitable oral care product to help prevent postoperative pneumonia. Breast milk, physiological saline, and sodium bicarbonate were used in this study. The results showed that the incidence of postoperative pneumonia was significantly lower in the breast milk oral care group than in the physiological saline oral care group and the sodium bicarbonate oral care group. The significantly reduced incidence of postoperative pneumonia would help shorten the duration of mechanical ventilation, length of ICU and hospital stay, and hospitalization cost. Using breast milk oral care could reduce postoperative pneumonia, and the reasons might be diverse. Breast milk is the most natural and safe natural food for infants during their growth, as it contains rich probiotics and nutrients and plays a vital role in increasing the immune function and self-resistance in infants. Besides, breast milk contains other bioactive substances, including immunoglobulin and a variety of cytokines, such as immunoglobulin A, lactoferrin, and breast milk oligosaccharides, which could play a barrier protection 
role in inhibiting pathogenic bacteria attached to the oral mucosa and preventing pathogens from invading the upper respiratory tract mucosa and causing postoperative pneumonia (25). Studies have shown that breast milk also contains many probiotics that are beneficial to prevent oral flora disorders and inhibit bacterial proliferation (26). Through a literature search, some studies showed that oral care intervention using breast milk could effectively prevent postoperative pneumonia $(27,28)$. Our results were consistent with the conclusions of these studies. We believe that breast milk oral care was beneficial, easy to implement, and cost-effective for the prognosis of those infants with prolonged endotracheal intubation and mechanical ventilation after cardiac surgery.

There are disadvantages to using breast milk for oral care. Once breast milk is contaminated, it is more likely to breed bacteria and fungi. Therefore, we should pay attention to obtaining, preserving, and using breast milk to ensure breast milk's cleanliness and hygiene. We should give health guidance to the family members and guide the mother to express milk correctly. We also should ensure the hygiene of breast milk during milking and transportation. Nurses should practice good hand hygiene in oral care to avoid cross-contamination.

There were still some limitations to this study. First, this was a study that used a single-center and small sample size. There was a specific deviation in case selection, and different centers and different research subjects might have different research results. Besides, the indexes adopted in this study were limited, and objective bacteriological indexes were missing, which might affect the conclusion's rigor. However, we still believe that our study had specific clinical significance and could serve as a reference for postoperative rehabilitation of such patients.

\section{Conclusions}

Compared with traditional physiological saline and sodium bicarbonate oral care, breast milk oral care is a simple, safe, economical, and easy-to-operate intervention that can reduce postoperative pneumonia incidence. Using breast milk oral care in infants with prolonged endotracheal intubation and mechanical ventilation after cardiac surgery is worthy of clinical application.

\section{Acknowledgments}

We positively acknowledged the participating researchers' contribution: Yi-Rong Zheng, Hong-Lin Wu, Ze-Wei Lin, Li-Wen Wang, Jing Wang, and Ling-Shan Yu.

Funding: None.

\section{Footnote}

Reporting Checklist: The authors have completed the CONSORT reporting checklist. Available at http://dx.doi. org/10.21037/tp-20-295

Data Sharing Statement: Available at http://dx.doi. org/10.21037/tp-20-295

Conflicts of Interest: All authors have completed the ICMJE uniform disclosure form (available at http://dx.doi. org/10.21037/tp-20-295). The authors have no conflicts of interest to declare.

Ethical Statement: The authors are accountable for all aspects of the work in ensuring that questions related to the accuracy or integrity of any part of the work are appropriately investigated and resolved. The ethics board of Fujian Medical University approved the study (No. 2020YJ181), and informed consent was taken from the patients' parents/ guardians. The study was conducted in accordance with the Declaration of Helsinki (as revised in 2013).

Open Access Statement: This is an Open Access article distributed in accordance with the Creative Commons Attribution-NonCommercial-NoDerivs 4.0 International License (CC BY-NC-ND 4.0), which permits the noncommercial replication and distribution of the article with the strict proviso that no changes or edits are made and the original work is properly cited (including links to both the formal publication through the relevant DOI and the license). See: https://creativecommons.org/licenses/by-nc-nd/4.0/.

\section{References}

1. Safdar N, Dezfulian C, Collard HR, et al. Clinical and economic consequences of postoperative pneumonia: a systematic review. Crit Care Med 2005;33:2184-93.

2. Patria MF, Chidini G, Ughi L, et al. postoperative pneumonia in an Italian pediatric intensive care unit: a prospective study. World J Pediatr 2013;9:365-8.

3. Ames NJ, Sulima P, Yates JM, et al. Effects of systematic oral care in critically ill patients: a multicenter study. Am J Crit Care 2011;20:e103-14. 
4. Needleman IG, Hirsch NP, Leemans M, et al. Randomized controlled trial of toothbrushing to reduce postoperative pneumonia pathogens and dental plaque in a critical care unit. J Clin Periodontol 2011;38:246-52.

5. Cason CL, Tyner T, Saunders S, et al. Nurses' implementation of guidelines for postoperative pneumonia from the Centers for Disease Control and Prevention. Am J Crit Care 2007;16:28-36.

6. Bonten MJ, Kollef MH, Hall JB. Risk factors for postoperative pneumonia: from epidemiology to patient management. Clin Infect Dis 2004;38:1141-9.

7. Stonecypher K. Postoperative pneumonia the importance of oral care intubated adults. Crit Care Nurs Q 2010;33:339-47.

8. Paju S, Scannapieco FA. Oral biofilms, peritonitis, and pulmonary infections. Oral Dis 2007;13:508-12.

9. Browne JA, Evans D, Christmas LA, et al. Pursuing excellence: development of an oral hygiene protocol for mechanically ventilated patients. Crit Care Nurs Q 2011;34:25-30.

10. Haghighi A, Shafipour V, Bagheri-Nesami M, et al. The impact of oral care on oral health status and prevention of postoperative pneumonia in critically ill patients. Aust Crit Care 2017;30:69-73.

11. Coppadoro A, Bittner E, Berra L. Novel preventive strategies for ventilator- associated pneumonia. Crit Care 2012;16:210.

12. Pneumatikos IA, Dragoumanis CK, Bouros DE. Postoperative pneumonia or endotracheal tube-associated pneumonia? An approach to the pathogenesis and preventive strategies emphasizing the importance of endotracheal tube. Anesthesiology 2009;110:673-80.

13. Roeleveld PP, Guijt D, Kuijper EJ, et al. Postoperative pneumonia in children after cardiac surgery in The Netherlands. Intensive Care Med 2011;37:1656-63.

14. Rello J, Koulenti D, Blot S, et al. Oral care practices in intensive care units: a survey of 59 European ICUs. Intensive Care Med 2007;33:1066-70.

15. Hutchins K, Karras G, Erwin E, et al. postoperative pneumonia and oral care: a successful quality improvement project. Am J Infect Control 2009;37:590-7.

16. Sona CS, Zack JE, Schallom ME, et al. The impact of a simple, low-cost oral care protocol on postoperative pneumonia rates in a surgical intensive care unit. J Intensive Care Med 2009;24:54-62.

17. Luo H, Qin G, Wang L, Ye Z, et al. Outcomes of Infant
Cardiac Surgery for Congenital Heart Disease Concomitant With Persistent Pneumonia: A Retrospective Cohort Study. J Cardiothorac Vasc Anesth 2019;33:428-32.

18. Hoffman JIE, Kaplan S. The incidence of congenital heart disease. J Am Coll Cardiol 2002;39:1890-900.

19. Garcia R, Jendresky L, Colbert L, et al. Reducing postoperative pneumonia through advanced oral-dental care: a 48-month study. Am J Crit Care 2009;18:523-32.

20. Roberts N, Moule P. Chlorhexidine and tooth-brushing as prevention strategies in reducing postoperative pneumonia rates. Nurs Crit Care 2011;16:295-302.

21. Scannapieco FA. Pneumonia in nonambulatory patients: the role of oral bacteria and oral. J Am Dent Assoc 2006;137:21S-25S.

22. Mori H, Hirasawa H, Oda S, et al. Oral care reduces incidence of postoperative pneumonia in ICU populations. Intensive Care Med 2006;32:230-6.

23. Messika J, La Combe B, Ricard JD. Oropharyngeal colonization: epidemiology, treatment and postoperative pneumonia prevention. Ann Transl Med 2018;6:426.

24. Hillier B, Wilson C, Chamberlain D, et al. Preventing postoperative pneumonia through oral care, product selection, and application method: a literature review. AACN Adv Crit Care 2013;24:38-58.

25. Meier P, Patel A, Esquerra-Zwiers A. Donor breast milk Update: Evidence, Mechanisms, and Priorities for Research and Practice. J Pediatr 2017;180:15-21.

26. Smilowitz JT, Totten SM, Huang J, et al. Breast milk secretory immunoglobulin a and lactoferrin $\mathrm{N}$-glycans are altered in women with gestational diabetes mellitus. J Nutr 2013;143:1906-12.

27. Rodriguez NA, Meier PP, Groer MW, et al. A pilot study to determine the safety and feasibility of oropharyngeal administration of own mother's colostrum to extremely lowbirth-weight infants. Adv Neonatal Care 2010;10:206-12.

28. Lee J, Kim HS, Jung YH, et al. Oropharyngeal colostrum administration in extremely premature infants: an RCT. Pediatrics 2015;135:e357-66.

Cite this article as: $\mathrm{Yu} \mathrm{XR}, \mathrm{Xu}$ N, Huang ST, Zhang QL, Wang ZC, Cao H, Chen Q. Effects of different oral care strategies on postoperative pneumonia in infants with mechanical ventilation after cardiac surgery: a prospective randomized controlled study. Transl Pediatr 2021;10(2):359-365. doi: 10.21037/tp-20-295 